\title{
Piriformis syndrome or true sciatica: are they the same or different?
}

\begin{abstract}
Sciatica is musculoskeletal pain sensed in the leg along the distribution of the sciatic nerve, sometimes accompanied by low back pain. While there are many causes of sciatica, piriformis syndrome is an often misdiagnosed cause of sciatica. The sciatic nerve courses inferior and anterior to the piriformis muscle as it exits the pelvis and courses down the posterior thigh and leg. Piriformis syndrome is caused by compression or irritation of the sciatic nerve due to spasm of the piriformis muscle. The most common presentation is pain in the posterior gluteal region that shoots down the leg in the area of the sciatic nerve. No definitive diagnostic test or universal diagnostic criteria exists for piriformis syndrome. First-line treatment consists of non-steroidal anti-inflammatory medications (NSAID). Surgery is often performed as a last resort once all other treatment options have been exhausted and other etiologies have been excluded. Piriformis syndrome is a diagnosis of exclusion, and often an underappreciated cause of sciatica.
\end{abstract}

Volume 9 Issue 3 - 2017

\author{
Nabil Ebraheim, Kyle Andrews, Briana \\ Stirling, Mina Tanios \\ Department of Orthopaedic Surgery, University of Toledo \\ Medical Center
}

\author{
Correspondence: Nabil Ebraheim, Department of \\ Orthopaedic Surgery, University of Toledo Medical Center, USA \\ Email nebraheim@aol.com
}

Received: May 22, 2017 | Published: November 01, 2017

\section{Introduction}

Sciatica is musculoskeletal pain sensed in the leg along the distribution of the sciatic nerve, which runs from the lower back, through the buttock, to the posterior thigh, and into the posterolateral lower leg and foot; sometimes accompanied by low back pain. Sciatica most often affects one side of the body and is often referred to as lumbar radiculopathy. Disc herniation is the most common cause, however sciatica is not a diagnosis, it is a symptom of an underlying condition. While there are many causes of sciatica, piriformis syndrome is an often misdiagnosed cause of sciatica, leg, or buttock pain. Piriformis syndrome is defined as a neuromuscular disorder attributed to the compression of the sciatic nerve around the buttock and hip causing sciatica. Piriformis syndrome is a diagnosis of exclusion, but often a hidden cause of sciatica.

\section{Anatomy}

The piriformis muscle is a muscle pair shaped like a flat pyramid which originates at the levels of S2-S4 on the anterolateral aspect of the sacrum. It exits the pelvic cavity by sliding through the greater sciatic notch and runs obliquely through the gluteal region and inserts on the posterosuperior aspect of the greater trochanter of the femur. In the gluteal region, the piriformis muscle is located deep to the gluteus maximus and superior to the obturator internus muscle tendon, which is accompanied by the gemelli muscles. The sciatic nerve courses inferior and anterior to the piriformis muscle as it exits the pelvis and courses down the posterior thigh and leg. As it exits inferior to piriformis, it becomes posterior to the gemelli and obturator internus (Figure 1). The sciatic nerve is composed of the tibial and peroneal divisions, which are usually bound together, but sometimes may divide as they pass the piriformis muscle. There are four types of anatomical variation of the sciatic nerve. Type 1 consists of the traditionally described anatomy, where the sciatic nerve passes below the piriformis muscle. Type 2 occurs when the sciatic nerve divisions pass through and below the piriformis muscle. In type 3 the nerve divisions pass through and above the piriformis. Lastly, type 4 is when the entire nerve passes superior to the piriformis. ${ }^{1}$

\section{Etiology}

There are multiple reasons patients may experience sciatica. Some common examples include lumbar disc herniation, lumbar spinal stenosis, epidural lipomatosis, and piriformis syndrome. Piriformis syndrome is caused by compression or irritation of the sciatic nerve due to spasm of the piriformis muscle. In most patients the exact cause of piriformis spasm is unknown. Muscle tightness, adhesions, and spasms are likely caused by overuse activities such as bicycling and other sporting activities. Pressure on the nerve can cause swelling, inflammation, and other symptoms of nerve irritation. Other causes of piriformis syndrome are thought of as anatomical anomalies, such as split piriformis, aberrations or vascular structures, or unusual sciatic nerve path.

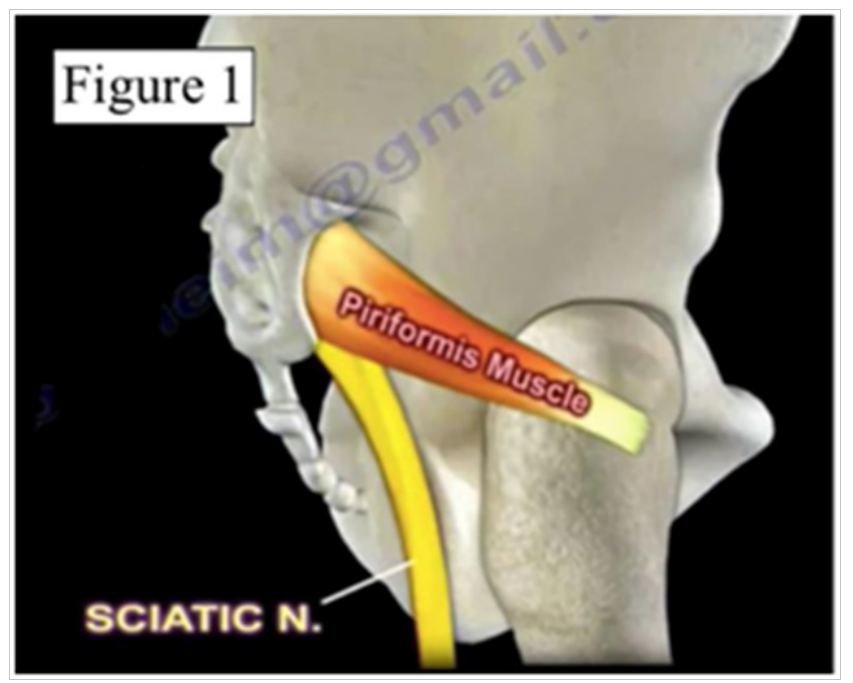

Figure I Depicts the most common anatomic relationship of the sciatic nerve exiting anterior to the piriformis muscle. ${ }^{5}$

\section{Clinical presentation}

The most common presentation is pain in the posterior gluteal region that shoots down the leg in the area of the sciatic nerve causing sciatica. The symptoms are characterized as burning, tingling and shooting pains. The pain is most often unilateral and radiates from the buttocks through the leg down towards the toes in a dermatomal pattern. Numbness and paresthesia may be present within the same distribution. Leg pain is usually greater than back pain, if present. The patient may also experience intense pain while sitting, squatting or climbing stairs. Tenderness to palpation in the area of the sciatic notch 
may be detected on exam, corresponding to the piriformis muscle ${ }^{2}$ (Figure 2).

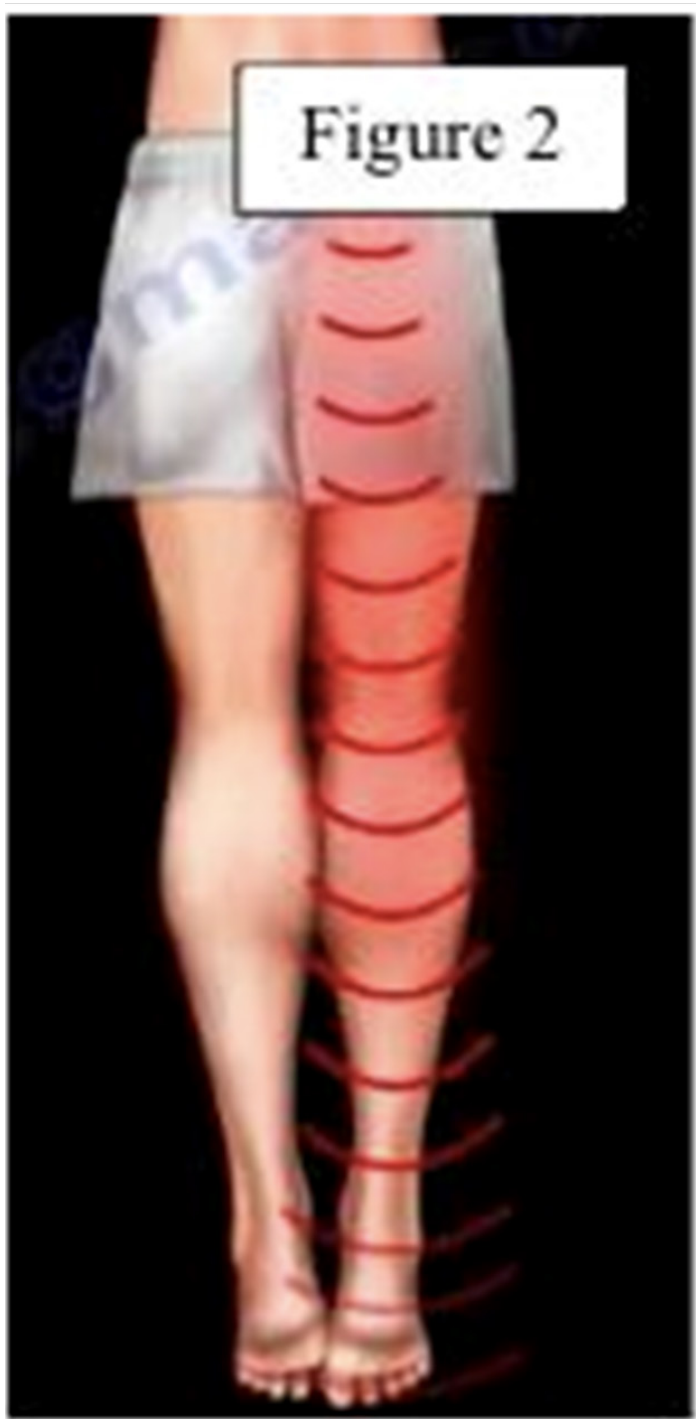

Figure 2 Showing the common distribution of symptoms of sciatic nerve irritation. $^{5}$

\section{Diagnosis}

No definitive diagnostic test or universal diagnostic criteria exists for piriformis syndrome. A high index of suspicion is needed for diagnosis. A thorough medical history and physical examination are essential, followed by diagnostic testing to differentiate piriformis syndrome from other causes of sciatica. An MRI could show an enlarged piriformis, anomalies of the vessels, and compression of the nerve. However, imaging studies are commonly performed to rule out other causes of sciatic nerve and gluteal pain. Physical examination techniques are often used to determine the underlying causes of sciatica. The FAIR test is a sensitive and specific test for piriformis syndrome. FAIR stands for flexion, adduction and internal rotation. The purpose of this test is to stretch the piriformis muscle compressing the sciatic nerve. To perform, have the patient lie with the affected side up, the examiner stabilizes the flexed hip and applies downward pressure to the knee to internally rotate and adduct the hip. Reproduction of the patient's symptoms is considered a positive test ${ }^{3}$ (Figure 3).

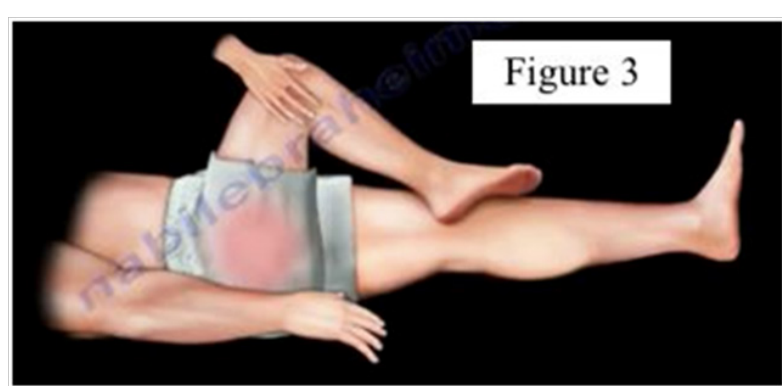

Figure 3 The FAIR test - a sensitive and specific test for piriformis syndrome. ${ }^{5}$

Lasegue's test, or straight leg raise, is performed to determine if a patient with sciatica has an underlying herniated disc. This is a passive test used to reproduce pain along the sciatic nerve distribution by flexing the hip while keeping the knee extended.

\section{Treatment}

First-line treatment consists of non-steroidal anti-inflammatory medications (NSAID), however continued use of NSAIDs can have adverse renal and gastrointestinal effects and the patient must be educated. Additional conservative measures include physical therapy using piriformis stretching and isometric strengthening. Physical therapists often use the FAIR maneuver as treatment. If these measures are unsuccessful, consider local nerve block injections under ultrasound or fluoroscopy guidance into the piriformis muscle, which is considered both therapeutic and diagnostic if the etiology remains unclear after initial evaluation. Injections may include local anesthetic and steroid or botulinum toxin (BTX). Surgery is often performed as a last resort once all other treatment options have been exhausted and other etiologies have been excluded. Surgery may include the release of the piriformis muscle from the greater trochanter with or without sciatic nerve neurolysis. ${ }^{4}$

\section{Summary}

Piriformis syndrome should be differentiated from other causes of sciatica. It is often an underappreciated diagnosis, but remains a diagnosis of exclusion. Sciatica should be evaluated by differentiating between spinal causes, such as compression of the nerve root, or compression of the sciatic nerve itself as in piriformis syndrome. The main cause of true sciatica is a lumbar disc herniation. Conservative treatment is often successful, but more invasive measures remain as treatment options. Patients should consult with their doctor before determining a course of treatment.

\section{Acknowledgements}

None.

\section{Conflicts of interest}

None.

\section{References}

1. Cass SP. Piriformis syndrome: a cause of nondiscogenic sciatica. Curr Sports Med Rep. 2015;14(1):41-44.

2. Jankovic D, Peng $P$, van Zundert A. Brief review: piriformis syndrome: etiology, diagnosis, and management. Can J Anaesth. 2013;60(10):1003-1012

3. Michel F, Decavel P, Toussirot E, et al. The piriformis muscle syndrome: an exploration of anatomical context, pathophysiological hypotheses and diagnostic criteria. Ann Phys Rehabil Med. 2013;56(4):300-311. 
4. Koes BW, van Tulder MW, Peul WC. Diagnosis and treatment of sciatica. BMJ. 2007;34(7607):1313-1337.

5. Ebraheim MN. Piriformis Syndrome or True Sciatica - Everything You Need To Know - Dr. Nabil Ebraheim. 2017.

6. Ropper AH, Zafonte RD. Sciatica. N Engl J Med. 2015;372(13):1240.
7. Papadopoulus EC, Khan SN. Piriformis syndrome and low back pain: a new classification and review of the literature. Orthop Clin North Am. 2004;35(1):65-71

8. Chou R. In the clinic. Low back pain. Ann Intern Med.2014;160(11):ITC6. 